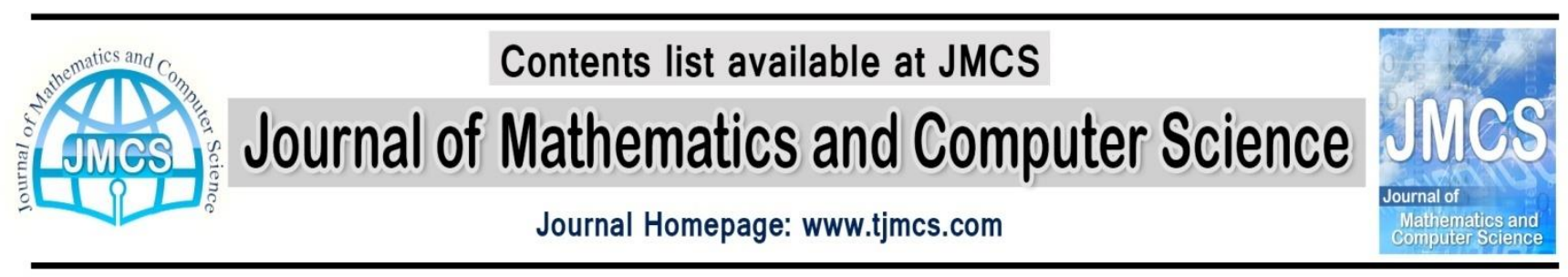

\title{
Conformal $h$-Vector-Change in Finsler Spaces
}

\author{
A. Taleshian ${ }^{1}$, D. M. Saghali ${ }^{2}$, S.A. Arabi \\ ${ }^{1}$ Department of Mathematics, University of Mazandaran, Babolsar, Iran, \\ taleshian@umz.ac.ir \\ ${ }^{2}$ Department of Mathematics, University of Mazandaran, Babolsar, Iran, \\ dordisaghali@yahoo.com \\ Article history: \\ Received May \\ Accepted June \\ Available online June
}

Abstract

We investigate what we call a conformal $h$-vector-change in Finsler spaces, namely

$$
F(x, y) \rightarrow \bar{F}(x, y)=e^{\sigma(x)} F(x, y)+\beta,
$$

where, $\sigma$ is a function of $x$ only, and $\beta(x, y):=b_{i}(x, y) y^{i}$, where $b_{i}:=b_{i}(x, y)$ is an $h$-vector. This change generalizes various types of changes: conformal changes, generalized Randers changes, Randers change. Under this change, we obtain the relationships between some tensors associated with $(M, F)$ and the corresponding tensors associated with $(M, \bar{F})$. Next, we express the conditions for more generalized $m$-th root metrics $\tilde{F}_{1}=$ $\sqrt{A_{1}^{\frac{2}{m_{1}}}+B_{1}}+C_{1}$ and $\tilde{F}_{2}=\sqrt{A_{2}^{\frac{2}{m_{2}}}+B_{2}}+C_{2}$, when is established conformal $h$-vector-change and $m_{1}, m_{2}$ are even numbers and other case $m_{1}, m_{2}$ even and odd numbers, respectively. Finally, we prove that under these conditions conformal $h$-vector-change in Finsler spaces reduces to conformal $\beta$-change in Finsler spaces.

Keywords: $m$-th root metric; more generalized $m$-th root metric; generalized Randers change.

\section{Introduction}

Let $(M, F)$ be a Finsler space, where $M$ is an $n$-dimensional differentiable manifold equipped with a fundamental function $F$. Given a function $\sigma$, the change

$$
\bar{F}(x, y) \rightarrow e^{\sigma(x)} F(x, y)
$$

is called a conformal change. The conformal theory of Finsler spaces has been initiated by M. S. Knebelman [1] in 1929 and has been deeply investigated by many authors: [2], [3], etc. For a differential one-form $\beta(x, y)=$ $b_{i}(x) y^{i}$ on $M$, G. Randers [4], in 1941, introduced a special Finsler space defined by the $\beta$-change 


$$
\bar{F}(x, y)=F(x, y)+\beta(x, y),
$$

where $F$ is Riemannian. The resulting space is a Finsler space. Randers metrics are among the simplest nonRiemannian Finsler metrics. M. Matsumoto [5], in 1974, studied Randers space in which $F$ is Finslerian. In 1980, H. Izumi [6] introduced the concept of an $h$-vector $b_{i}$, while studying the conformal transformation of Finsler spaces. Then, instead of the function $b_{i}$ of coordinates $x^{i}$ only, we will use the $h$-vector $b_{i}(x, y)$ and define the generalized Randers change

$$
\bar{F}(x, y)=F(x, y)+b_{i}(x, y) y^{i} .
$$

We can find some results regarding the generalized Randers change in B. N. Prasad [7] and M. Gupta and P. Pandey [8]. In 2008, S. Abed ([9], [10]) introduced the transformation

$$
\bar{F}(x, y)=e^{\sigma(x)} F(x, y)+\beta(x, y)
$$

Moreover, he established the relationships between some important tensors associated with $(M, F)$ and the corresponding tensors associated with $(M, \bar{F})$. He also studied some invariant and $\sigma$-invariant properties and obtained a relationship between the Cartan connection associated with $(M, F)$ and the transformed Cartan connection associated with $(M, \bar{F})$.

In 1979, Shimada [11] introduced the $m$-th root metric on the differentiable manifold $M$ defined as:

$$
F=\sqrt[m]{a_{i_{1} i_{2} \ldots i_{m}}(x) y^{i_{1}} y^{i_{2}} \ldots y^{i_{m}}}
$$

where the coefficients $a_{i_{1} i_{2} \ldots i_{m}}$ are the components of symmetric covariant tensor field of order $(0, m)$ being the functions of positional co-ordinates only. Since then various geometers such as [12], [13] etc. have explored the theory of $m$-th root metric and studied its transformations. There exist the following important two classes of Finsler metrics,

$$
\begin{aligned}
& \bar{F}=\sqrt{A^{\frac{2}{m}}+B}, \\
& \tilde{F}=\sqrt{A^{\frac{2}{m}}+B}+C,
\end{aligned}
$$

where $A=a_{i_{1} i_{2} \ldots i_{m}}(x) y^{i_{1}} y^{i_{2}} \ldots y^{i_{m}}, B=b_{i j}(x) y^{i} y^{j}$ and $C=c_{k}(x) y^{k}$, that is one 1 -form. This forms are called a generalized $m$-th root metric and more general generalized $m$-th root metric, respectively. Obviously, $\tilde{F}$ is not reversible Finsler metric and is Randers change of generalized $m$-th root metric $\bar{F}$. In [14], the authors have studied the geometric properties of locally projectively flat $m$-th root in the form $F=\sqrt[m]{A}$ and generalized $m$-th root in the form $\bar{F}=\sqrt{A^{\frac{2}{m}}+B}$. In [15], Tayebi-Najafi characterize locally dually flat and Antonelli $m$-th root metrics. They prove that every $m$-th root metric of isotropic mean Berwald curvature (resp., isotopic Landsberg curvature) reduces to a weakly Berwald metric (resp., Landsberg metric). They show that $m$-th root metric with almost vanishing $\mathrm{H}$-curvature has vanishing H-curvature [16]. In [17], the authors expresses a necessary and sufficient condition for the metric $\bar{F}=\sqrt{A^{\frac{2}{m}}+B}$ that be locally dually flat. In [18], the authors have studied Berwald $m$-th root metrics. Y. Yu and Y. You show that an $m$-th root Einstein Finsler metric is Ricci-flat [19]. 
In this paper, we construct a theory which generalizes all the above mentioned changes. In fact, we consider a change of the form

$$
\bar{F}(x, y) \rightarrow e^{\sigma(x)} F(x, y)+\beta(x, y)
$$

where, $\sigma$ is a function of $x$ only, and $\beta(x, y):=b_{i}(x, y) y^{i}$, where $b_{i}:=b_{i}(x, y)$ is an $h$-vector on $(M, F)$, which we call conformal h-vector-change. This change generalizes various types of changes. When $\beta=0$, it is reduces to a conformal change defined in (1.1). When $\sigma=0$, it reduces to a generalized Randers change defined in (1.3). when $\beta$ reduces to a one-form $b_{i}(x)$, then it reduces to a conformal $\beta$-change defined in (1.4). when $\sigma=0$ and $\beta$ reduces to a one-form $b_{i}(x)$, then it reduces to a Randers $\beta$-change defined in (1.2). Thus the study of this new class of Finsler spaces will enhance our understanding of the geometric meaning of conformal changes and Randers changes. Under this change, we obtain the relationships between components of the more generalized $m$-th root metrics $\tilde{F}_{1}=\sqrt{A_{1}^{\frac{2}{m_{1}}}+B_{1}}+C_{1}$ and $\tilde{F}_{2}=\sqrt{A_{2}^{\frac{2}{m_{2}}}+B_{2}}+C_{2}$, when is established conformal h-vector-change and $m_{1}, m_{2}$ are even numbers and other case $m_{1}, m_{2}$ even and odd numbers, respectively. As well As, we prove that under these conditions conformal $h$-vector-change in Finsler space with more generalized $m$-th root metric reduces to conformal $\beta$-change in Finsler space with more generalized $m$-th root metric.

Theorem 1. Let $\tilde{F}_{1}=\sqrt{A_{1}^{\frac{2}{m_{1}}}+B_{1}}+C_{1}$ and $\tilde{F}_{2}=\sqrt{A_{2} \frac{2}{m_{2}}+B_{2}}+C_{2}$ are two more generalized $m$-th root metrics on an open subset $U \subset R^{n}$. Suppose that $m_{1}, m_{2}$ are even numbers and $m_{1}, m_{2}>2$. If $\tilde{F}_{1}$ is conformal $h$ vector-change of $\tilde{F}_{2}$, then $\tilde{F}_{1}$ reduces to a conformal $\beta$-change of $\tilde{F}_{2}$.

Theorem 2. Let $\tilde{F}_{1}=\sqrt{A_{1}^{\frac{2}{m_{1}}}+B_{1}}+C_{1}$ and $\tilde{F}_{2}=\sqrt{A_{2}^{\frac{2}{m_{2}}}+B_{2}}+C_{2}$ are two more generalized $m$-th root metrics on an open subset $U \subset R^{n}$. Suppose that $m_{1}, m_{2}$ are even and odd numbers, respectively. If $\tilde{F}_{1}$ is conformal $h$ vector-change of $\tilde{F}_{2}$, then $\tilde{F}_{1}$ reduces to a conformal $\beta$-change of $\tilde{F}_{2}$.

Throughout the present paper, $\left(x^{i}\right)$ denotes the coordinates of a point of the base manifold $M$ and $\left(y^{i}\right)$ the supporting element $\left(\dot{x}^{i}\right)$. As well as, in overall this paper,

$$
\begin{aligned}
& A_{1}=a_{i_{1} i_{2} \ldots i_{m_{1}}}(x) y^{i_{1}} y^{i_{2}} \ldots y^{i_{m_{1}}}, \\
& A_{2}=\bar{a}_{i_{1} i_{2} \ldots i_{m_{2}}}(x) y^{i_{1}} y^{i_{2}} \ldots y^{i_{m_{2}}}, \\
& \mathrm{~B}_{1}=b_{i j}(x) y^{i} y^{j}, \mathrm{~B}_{2}=\bar{b}_{i j}(x) y^{i} y^{j}, \\
& \mathrm{C}_{1}=c_{k}(x) y^{k}, \mathrm{C}_{2}=\bar{c}_{k}(x) y^{k},
\end{aligned}
$$

and $m_{1}, m_{2}$ are belongs to natural numbers, and $b_{i}(x,-y)=b_{i}(x, y)$.

\section{Preliminaries}

Let $M$ be an $n$-dimensional $C^{\infty}$ manifold. Denote by $T_{x} M$ the tangent space at $x \in M$, and by $T M=\cup_{x \in M} T_{x} M$, the tangent bundle of $M$. A Finsler metric on $M$ is a function $F: T M \rightarrow[0, \infty)$ which has the following 
properties: (i) $F$ is $C^{\infty}$ on the slit tangent bundle $T M_{0}=T M-\{0\}$; (ii) $F$ is positively 1-homogeneous on the fibers of the tangent bundle $T M$; (iii) for each $y \in T_{x} M$, the following quadratic form $g_{y}$ on $T_{x} M$ is positive definite,

$$
\boldsymbol{g}_{y}(u, v):=\left.\frac{1}{2} \frac{\partial^{2}}{\partial s \partial t}\left[F^{2}(y+s u+t v)\right]\right|_{s, t=0}, \quad u, v \in T_{x} M
$$

Let $x \in M$ and $F_{x}:=\left.F\right|_{T_{x} M}$. For $y \in T_{x} M_{0}$, define $C_{y}: T_{x} M \otimes T_{x} M \otimes T_{x} M \rightarrow R$ by

$$
\boldsymbol{C}_{y}(u, v, w):=\left.\frac{1}{2} \frac{d}{d t}\left[g_{y+t w}(u, v)\right]\right|_{t=0}, u, v, w \in T_{x} M .
$$

The family $\boldsymbol{C}:=\left\{\boldsymbol{C}_{y}\right\}_{y \in T_{x} M_{0}}$ is called the Cartan torsion. It is well known that $\boldsymbol{C}=\mathbf{0}$ if and only if $F$ is Riemannian [20]. The $h$-vector $b_{i}$ is $v$-covariant constant with respect to the Cartan connection and satisfies

$$
F C_{i, j}^{h} b_{h}=\rho h_{i j}, \rho \neq 0,
$$

where, $C_{i j}^{h}:=g^{r h} C_{h j k}$ (where, $g^{r h}$ and $C_{h j k}$ are components inverse of $g_{r h}$ and Cartan torsion, respectively.) is the Cartan's $\boldsymbol{C}$-tensor, $h_{i j}$ is the angular metric tensor and $\rho$ is given by

$$
\rho=\frac{F C^{i} b_{i}}{(n-1)}
$$

where, $C^{i}$ is the torsion vector $C_{j k}^{i} g^{j k}$. Then, we have

$$
\dot{\partial}_{j} b_{i}=\frac{\rho h_{i j}}{F}=\rho F_{y^{i} y^{j}} \neq 0,
$$

Where, $\dot{\partial}_{j}=\frac{\partial}{\partial y^{j}}$ and $\rho$ is independent of directional arguments.

For an $h$-vector we have the following [6]:

Lemma 1. If $b_{i}$ is an $h$-vector then function $\rho \bar{F}_{y^{i}}=b_{i}-\rho F_{y^{i}}$ are independent of $y$.

Lemma 2. The magnitude $b$ of an $h$-vector $b_{i}$ is independent of $y$.

\section{Cartan's connection of the conformal $h$-vector-change}

Let $b_{i}$ is a vector field in the Finsler space $(M, F)$. If we denote $b_{i} y^{i}$ as $\beta$ then indicatory property of $h_{i j}$ yield $\dot{\partial}_{j} \beta_{i}=b_{i}$. Throughout the paper we shall use the notations

$$
\begin{gathered}
l_{i}:=\dot{\partial}_{i} F=\frac{\partial F}{\partial y^{i}} \\
l_{i j}:=\dot{\partial}_{i} \dot{\partial}_{j} F, l_{i j k}:=\dot{\partial}_{i} \dot{\partial}_{j} \dot{\partial}_{k} F \text { and etc. }
\end{gathered}
$$

Now, the quantities and operations referring to $c$ are indicated by putting - Thus, from (1.7) we get 


$$
\bar{l}_{i}(x, y)=e^{\sigma(x)} l_{i}(x, y)+b_{i}(x, y),
$$

Then from (2.5) and (3.1), we get

$$
\bar{l}_{i j}(x, y)=e^{\sigma(x)} l_{i j}(x, y)+\rho F_{y^{i} y^{j}}=\left(e^{\sigma(x)}+\rho\right) l_{i j}(x, y),
$$

and so on. The angular metric tensor $\bar{h}_{i j}$ is given in terms of $h_{i j}$ by

$$
\bar{h}_{i j}=\bar{F} \bar{l}_{i j}=\bar{F}\left(e^{\sigma}+\rho\right) l_{i j}=\tau h_{i j}, \tau=\left(e^{\sigma}+\rho\right) \frac{\bar{F}}{F}
$$

Lemma 3. Under a conformal $h$-vector-change, the angular metric $\bar{h}_{i j}$ of the metric $h_{i j}$ is given by (3.4).

As $h_{i j}=g_{i j}-l_{i} l_{j}$, equation (3.4) give us a relation between the fundamental tensors $g_{i j}, \bar{g}_{i j}$ :

$$
\bar{g}_{i j}=\tau g_{i j}+\left(e^{2 \sigma}-\rho\right) l_{i} l_{j}+e^{\sigma} l_{i} b_{j}+e^{\sigma} l_{j} b_{i}+b_{i} b_{j},
$$

Lemma 4. Under a conformal $h$-vector-change, the metric tensor $\bar{g}_{i j}$ of the metric $g_{i j}$ is given by (3.5).

From the equation

$$
\dot{\partial}_{k} h_{i j}=2 c_{i j k}-F^{-1}\left(l_{i} h_{j k}+l_{j} h_{i k}\right),
$$

we obtain

$$
l_{i j k}=2 F^{-1} c_{i j k}-F^{-2}\left(l_{i} h_{j k}+l_{j} h_{k i}+l_{k} h_{i j}\right),
$$

where, we put $c_{i j k}=\frac{\dot{\partial}_{k} g_{i j}}{2}$. Therefore, (3.3) is rewritten as the relation between $c_{i j k}$ and $\bar{c}_{i j k}$ :

$$
\bar{c}_{i j k}=\tau\left[c_{i j k}+\frac{1}{2 \bar{F}} h_{i j k}\right]
$$

where,

$$
\begin{gathered}
h_{i j k}=h_{i j} m_{k}+h_{j k} m_{i}+h_{k i} m_{j}, \\
m_{i}=b_{i}-\left(\frac{\beta}{F}\right) l_{i} .
\end{gathered}
$$

Theorem 3. Under a conformal $h$-vector-change, the $h(h v)$-torsion tensor $\bar{c}_{i j k}$ of the metric $c_{i j k}$ is given by (3.8). 


\subsection{Proof of theorem 1}

In this section, we prove Theorem 1 . To prove it, we need the following.

Theorem 4. Let $\tilde{F}_{1}=\sqrt{A_{1}^{\frac{2}{m_{1}}}+B_{1}}+C_{1}$ and $\tilde{F}_{2}=\sqrt{A_{2}^{\frac{2}{m_{2}}}+B_{2}}+C_{2}$ are two more generalized $m$-th root metrics on an open subset $U \subset R^{n}$. Suppose that $m_{1}, m_{2}$ are even numbers with $m_{1} \neq m_{2}$ and $m_{1}>m_{2}>2$. If $\tilde{F}_{1}$ is conformal $h$-vector-change of $\tilde{F}_{2}$, then $A_{1}= \pm\left(e^{m_{2} \sigma(x)} A_{2}\right)^{\frac{m_{1}}{m_{2}}}, B_{1}=e^{2 \sigma(x)} B_{2}$ and $C_{1}=e^{\sigma(x)} C_{2}+b_{i}(x, y) y^{i}$.

Proof. Under the assumption, we have

$$
\sqrt{A_{1}^{\frac{2}{m_{1}}}+B_{1}}+C_{1}=e^{\sigma(x)}\left(\sqrt{A_{2}^{\frac{2}{m_{2}}}+B_{2}}+C_{2}\right)+b_{i}(x, y) y^{i} \text {. }
$$

By putting (-y) instead of (y) in (4.1), we have

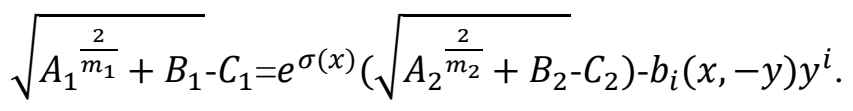

Summing sides the two equations (4.1) and (4.2), we have

$$
A_{1}^{\frac{2}{m_{1}}}+B_{1}=e^{2 \sigma(x)} A_{2}^{\frac{2}{m_{2}}}+e^{2 \sigma(x)} B_{2}
$$

Consequently, because of $m_{1}>m_{2}>2$, we get the proof.

Corollary 5. Let $\tilde{F}_{1}=\sqrt{A_{1}^{\frac{2}{m_{1}}}+B_{1}}+C_{1}$ and $\tilde{F}_{2}=\sqrt{A_{2}^{\frac{2}{m_{2}}}+B_{2}}+C_{2}$ are two more generalized $m$-th root metrics on an open subset $U \subset R^{n}$. Suppose that $m_{1}, m_{2}$ are even numbers with $m_{1}=m_{2}$ and $m_{1}\left(\right.$ or $\left.m_{2}\right)>2$. If $\tilde{F}_{1}$ is conformal $h$-vector-change of $\widetilde{F}_{2}$, then $A_{1}= \pm e^{m_{1} \sigma(x)} A_{2}\left(\right.$ or $\left.A_{1}= \pm e^{m_{2} \sigma(x)} A_{2}\right), B_{1}=e^{2 \sigma(x)} B_{2}$ and $C_{1}=e^{\sigma(x)} C_{2}+b_{i}(x, y) y^{i}$.

Proof. From (4.3) for $m_{1}=m_{2}$, one easily we get the proof.

Theorem 6. Let $\tilde{F}_{1}=\sqrt{A_{1}^{\frac{2}{m_{1}}}+B_{1}}+C_{1}$ and $\tilde{F}_{2}=\sqrt{A_{2}^{\frac{2}{m_{2}}}+B_{2}}+C_{2}$ are two more generalized $m$-th root metrics on an open subset $U \subset R^{n}$. Suppose that $m_{1}, m_{2}$ are even numbers with $m_{1} \neq m_{2}$ and $m_{1}>m_{2}>2$. If $\tilde{F}_{1}$ is conformal $h$-vector-change of $\tilde{F}_{2}$, then $\tilde{F}_{1}$ reduces to a conformal $\beta$-change of $\tilde{F}_{2}$.

Proof. Suppose that $\tilde{F}_{1}$ is conformal $h$-vector-change of $\tilde{F}_{2}$. Then

$$
C_{1}=e^{\sigma(x)} C_{2}+b_{i}(x, y) y^{i} .
$$

Differentiating (4.4) with respect to $y^{k}$, we have 


$$
c_{k}(x)=e^{\sigma(x)} \bar{c}_{k}(x)+b_{k}(x, y)
$$

Then

$$
\dot{\partial}_{j} b_{k}(x, y)=0
$$

Therefore, $b_{i}$ are functions of coordinates $x^{i}$ alone and from (2.5), $b_{i}$ is not a $h$-vector. Then we get the proof.

Similar to theorem 7, we have the following result:

Corollary 7. Let $\tilde{F}_{1}=\sqrt{A_{1}^{\frac{2}{m_{1}}}+B_{1}}+C_{1}$ and $\tilde{F}_{2}=\sqrt{A_{2}^{\frac{2}{m_{2}}}+B_{2}}+C_{2}$ are two more generalized $m$-th root metrics on an open subset $U \subset R^{n}$. Suppose that $m_{1}, m_{2}$ are even numbers with $m_{1} \neq m_{2}$ and $m_{1}>m_{2}>2$. If $\tilde{F}_{1}$ is conformal $h$-vector-change of $\tilde{F}_{2}$, then $\tilde{F}_{1}$ reduces to a conformal $\beta$-change of $\tilde{F}_{2}$.

Proof of theorem 1. Using theorem 7 and corollary 8, we get the proof.

\subsection{Proof of theorem 2}

In this section, we prove theorem 2 . To prove it, we need the following.

Theorem 8. Let $\tilde{F}_{1}=\sqrt{A_{1}^{\frac{2}{m_{1}}}+B_{1}}+C_{1}$ and $\tilde{F}_{2}=\sqrt{A_{2}^{\frac{2}{m_{2}}}+B_{2}}+C_{2}$ are two more generalized $m$-th root metrics on an open subset $U \subset R^{n}$. Suppose that $m_{1}, m_{2}$ are even and odd numbers, respectively. If $\tilde{F}_{1}$ is conformal $h$ vector-change of $\tilde{F}_{2}$, then

$$
\text { If } B_{1}=e^{2 \sigma(x)} B_{2} \text {, then } A_{1}= \pm \sqrt[\frac{m_{1}}{2}]{\frac{1}{2}\left(-B_{1} \pm \sqrt{\left(B_{1}\right)^{2}-\left(e^{m_{2} \sigma(x)} A_{2}\right)^{\frac{4}{m_{2}}}}\right.} \text { and } C_{1}=e^{\sigma(x)} C_{2}+b_{i}(x, y) y^{i} \text {. }
$$

$$
\text { If } B_{1} \neq e^{2 \sigma(x)} B_{2} \text {, then } A_{1}=
$$

$$
\begin{aligned}
& \pm \sqrt[\frac{m_{1}}{2}]{\frac{1}{2}\left(e^{2 \sigma(x)} B_{2}-2 B_{1} \pm \sqrt{\left(2 B_{1}-e^{2 \sigma(x)} B_{2}\right)^{2}-4\left(\left(B_{1}\right)^{2}-e^{2 \sigma(x)} B_{1} B_{2}+\frac{1}{4}\left(e^{m_{2} \sigma(x)} A_{2}\right)^{\frac{4}{m_{2}}}\right.}\right)} \text { and } \\
& C_{1}=e^{\sigma(x)} C_{2}+b_{i}(x, y) y^{i} .
\end{aligned}
$$

Proof. Under the assumption, we have

$$
\sqrt{A_{1}^{\frac{2}{m_{1}}}+B_{1}}+C_{1}=e^{\sigma(x)}\left(\sqrt{A_{2}^{\frac{2}{m_{2}}}+B_{2}}+C_{2}\right)+b_{i}(x, y) y^{i}
$$

By putting (-y) instead of (y) in (4.7), we have 


$$
\sqrt{A_{1}^{\frac{2}{m_{1}}}+B_{1}}-C_{1}=e^{\sigma(x)}\left(\sqrt{-A_{2}^{\frac{2}{m_{2}}}+B_{2}}-C_{2}\right)-b_{i}(x,-y) y^{i}
$$

Summing sides the two equations (4.7) and (4.8), we have

$$
2 \sqrt{A_{1}^{\frac{2}{m_{1}}}+B_{1}}=e^{\sigma(x)}\left(\sqrt{A_{2}^{\frac{2}{m_{2}}}+B_{2}}+\sqrt{-A_{2}^{\frac{2}{m_{2}}}+B_{2}}\right) .
$$

Thus

$$
4 A_{1}^{\frac{4}{m_{1}}}+4\left(B_{1}\right)^{2}+e^{4 \sigma(x)}\left(B_{2}\right)^{2}-4 e^{2 \sigma(x)} B_{1} B_{2}+4 A_{1}^{\frac{2}{m_{1}}}\left(2 B_{1}-e^{2 \sigma(x)} B_{2}\right)=\left(e^{2 \sigma(x)} B_{2}\right)^{2}-\left(e^{m_{2} \sigma(x)} A_{2}\right)^{\frac{4}{m_{2}}} .
$$

Now, if $B_{1}=e^{2 \sigma(x)} B_{2}$, then from (4.10) we have

$$
4 A_{1}^{\frac{4}{m_{1}}}+4 B_{1} A_{1}^{\frac{2}{m_{1}}}+\left(e^{m_{2} \sigma(x)} A_{2}\right)^{\frac{4}{m_{2}}}=0 .
$$

Consequently, we get the proof (i). If $B_{1} \neq e^{2 \sigma(x)} B_{2}$ then from (4.10), we get the proof of (ii).

Proof of theorem 2. Suppose that $\tilde{F}_{1}$ is conformal $h$-vector-change of $\tilde{F}_{2}$. Then from theorem 8 , we have

$$
C_{1}=e^{\sigma(x)} C_{2}+b_{i}(x, y) y^{i}
$$

Differentiating (4.12) with respect to $y^{k}$, we have

$$
c_{k}(x)=e^{\sigma(x)} \bar{c}_{k}(x)+b_{k}(x, y) .
$$

Then

$$
\dot{\partial}_{j} b_{k}(x, y)=0
$$

Therefore, $b_{i}$ are functions of coordinates $x^{i}$ alone and from (2.5), $b_{i}$ is not a $h$-vector. Then we get the proof.

\section{Acknowledgments}

The author D. M. Saghali wishes to express here his sincere gratitude to Dr. M. Rafie-rad for invaluable suggestion and encouragement. 


\section{References}

1. M. S. Knebelman, Conformal geometry of generalized metric spaces, Proc.nat.Acad. Sci.USA, 15(1929):3341 and 376-379.

2. M. Hashiguchi, On conformal transformation of Finsler metric, J. Math. Kyoto Univ., 16 (1976):25-50.

3. M. Kitayama, Geometry of transformations of Finsler metrics, Hokkaido University of Education, Kushiro Campus. Japan, (2000).

4. G. Randers, On the asymmetrical metric in the four-space of general relativity, Phys. Rev. (2) 59 (1941):195-199.

5. M. Matsumoto, On Finsler spaces with Randers metric and special forms of important tensors, J. Math. Kyoto Univ., 14 (1974):477-498.

6. H. Izumi, Conformal transformations of Finsler spaces, Tensor, N. S., 31 (1977):33-41.

7. B. N. Prasad, On the torsion tensors $R_{h j k}$ and $P_{h j k}$ of Finsler spaces with a metric $d s=\left(g_{i j}(d x) d x^{i} d x^{j}\right)^{\frac{1}{2}}+$ $b_{i}(x, y) d x^{i}$, Indian J. Pure Appl. Math., 21 (1990):27-39.

8. M. K. Gupta and P. N. Pandey, On hypersurface of a Finsler space with a special metric, Acta Math. Hungar., 120 (2008):165-177.

9. S. H. Abed, Conformal $\beta$-changes in Finsler spaces. Proc. Math. Phys. Soc. Egypt, 86 (2008):79-89.

10. S. H. Abed, Cartan connection associated with a \$lbeta \$-conformal change in Finsler geometry. Tensor, $N$. S., 70 (2008): 146-158.

11. H. Shimada, On Finsler spaces with metric $L=\sqrt[m]{a_{i_{1} i_{2} \ldots i_{m}} y^{i_{1}} y^{i_{2}} \ldots y^{i_{m}}}$, Tensor(N.S), 33 (1979):365-372.

12. B. N. Prasad and A. K. Dwivedi, On conformal transformation of Finsler spaces with $m$-th root metric, Indian J. pure appl. Math., 33(6) (2002):789-796.

13. A. Srivastava and P. Arora, Kropina change of mth root metric and its conformal transformation, Bull. of Calcutta Mathematical Society, 103(3) (2011).

14. S. Zhang. D. Zu. B. Li, projected flat $m$-th root Finsler metrics, J. Math. Ningbo Univ., 24 (3), (2010).

15. A. Tayebi, B. Najafi, On $m$-th root Finsler metrics. Geometry and Physics, (2011), 61:1479-1484.

16. A. Tayebi, B. Najafi, On $m$-th root metrics with special curvature properties. C. R. Acad. Sci, Ser. I, 349 (2011):691-693.

17. A. Tayebi, E. Peyghan, M. S. Nia, On generalized $m$-th root Finsler metrics. Linear Algebra Appl, ), 437 (2012):675-683.

18. D. Zu, S. Zhang and B. Li, On Berwald $m$-th root Finsler metrics, Publ. Math. Debercen, 4994 (2012):1-9.

19. Y. Yu, Y. You, On Einstein $m$-th root metrics. Differential Geometry and its Applications, 28, (2010):290294.

20. Z. Shen, Differential geometry of spray and Finsler spaces, Kluwer Academic Publishers, Dordrecht, (2001). 